\title{
Polyacrylamide gels with selective recognition of the tetrameric molecular form of human growth hormone
}

\author{
R. Kublickas ${ }^{1 *}$, C. Werner ${ }^{2}$, B. Voit $^{2}$ \\ ${ }^{1}$ Kaunas University of Technology, Department of Food Science and Technology, Radvilenu pl. 19, LT-50254 Kaunas, \\ Lithuania \\ ${ }^{2}$ Leibniz Institute of Polymer Research Dresden, Hohe Strasse 6, 01069 Dresden, Germany
}

Received 27 December 2016; accepted in revised form 6 March 2017

\begin{abstract}
Networks of polyacrylamide were studied for the possibility of imprinting of the oligomeric form of human growth hormone. The tetrameric molecular form of human growth hormone was molecularly imprinted for the first time. The results show that approximately $50-70 \%(\mathrm{w} / \mathrm{w})$ of the templates (depending on polymerization conditions) could be extracted from the molecularly imprinted acrylamide polymers. The resulting 'gel antibodies' against this form of human growth hormone in the form of granules of polyacrylamide were compared with granules of non-imprinted polymer. The selectivity of the artificial gel antibodies was studied. Investigation of the binding to imprinted polymer of the template hormone, other molecular forms of the hormone and other proteins shows the selectivity of the developed artificial gel antibodies.
\end{abstract}

Keywords: polymer gels, molecularly imprinted polymers, human growth hormone

\section{Introduction}

Human growth hormone $(\mathrm{hGH})$ like many bioactive substances, is a heterogeneous polypeptide [1]. This hormone plays an important role in somatic growth through its effects on the metabolism of proteins, carbohydrates, and lipids. Growth hormone with different biological activities occurs in the human body as several structural isoforms. The main molecular form is the so-called monomeric hGH with a molecular weight of $22 \mathrm{kDa}$, consisting of 191 amino acids. However, there are also molecular forms (isoforms) consisting of two or more (up to five) monomeric hGH units and it is very difficult to separate the usually obtained mixtures. There are no natural antibodies specific enough for recognizing these differently sized forms of the hormone.

Molecular imprinting is a recent promising technique for the fabrication of biomimetic polymeric recognition sites with a selective affinity for a target molecule (such as a drug, protein or biomacromolecule), which is attracting rapidly increasing interest and many potential applications in the pharmaceutical and biotechnological field [2-8]. Imprinted materials may constitute an alternative to natural recognition elements. In molecular imprinting, recognition sites are tailor-made in situ by self-assembly of suitable monomers and the templates followed by copolymerization with cross-linkers to form a polymer network in the presence of the template (Figure 1). The templates are subsequently washed from the molecularly imprinted polymer (MIP), leaving recognition sites complementary in the positioning of functional groups and in shape. The method creates materials resembling the binding sites of receptors and antibodies.

The polymerization reaction usually occurs by free radical initiation [2-12]. Numerous studies have been conducted to investigate different initiation mechanisms for MIP synthesis, thermal, photo-induced,

$\overline{{ }^{*} \text { Corresponding author, e-mail: rimantas.kublickas@ } @ \text { ktu.lt }}$

(C) BME-PT 


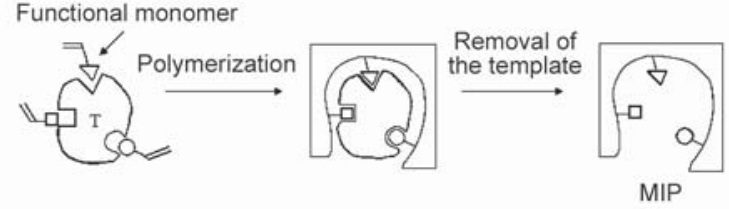

Figure 1. General scheme of molecular imprinting technology (T stands for template)

electrochemical or microwave-assisted methods were employed [7]. Polymerization at low temperatures provides for the most stable noncovalent interactions between functional monomer and template during the polymerization reaction. Several studies have shown that polymerization of MIP at lower temperatures forms polymers with greater selectivity versus polymers made at elevated temperatures [7]. Polymerization of MIP at lower temperatures are also important to prevent side reaction of the molecularly imprinted polymer and template molecule that can lead to covalent binding of the template to the MIP $[7,8,13]$. In case of thermal initiation in aqueous solutions (especially in case of the synthesis of protein imprinted hydrogels) a redox initiation system usually was used, including potassium or ammonium persulfate as an initiator and $N, N, N^{\prime}, N^{\prime}$-tetramethylethylene-diamine (TEMED) as accelerator [4, 7, 9-17]. TEMED accelerates the rate of formation of free radicals from persulfate and this initiation system it is very suitable in case of thermal initiated polymerization at lower temperatures.

The template must remain stable under the reaction conditions. Any change in conformation of the template could lead to incorrectly formed imprinted sites [18]. If a thermal initiation is chosen, the template should not degrade under the temperatures of polymerization.

Therefore the imprinting of macromolecules is a challenging research field [7]. Conventional molecular imprinting technology allows the synthesis in organic solvents of MIPs selective toward relatively low molecular weight compounds. Proteins mainly work in aqueous solutions in biological systems and many researchers have attempted to find the optimal conditions in protein imprinting [8]. Synthesis in aqueous media of chemically and mechanically stable MIPs that can recognize biomolecules such as peptides and proteins is still a great challenge [8]. Stellan Hjertén for the first time successfully synthesized highly selective artificial gel antibodies specific against proteins using acrylamide and methylene bisacrylamide as monomer and crosslinker respectively and aqueous buffer as a solvent by the molecular imprinting technique [9]. The method was to prepare the gel granules in the presence of a protein of interest $[9,10]$, where the protein was mixed and polymerized with a monomer solution (a buffer containing acrylamide, methylene bisacrylamide, TEMED and ammonium persulfate). It was shown that human growth hormone and some other proteins can also be adsorbed specifically on crosslinked polyacrylamide gel particles prepared by Hjertén's method using entrapment and molecular imprinting [10].

Some human peptide hormones and other larger peptides were successfully molecularly imprinted in aqueous media and show sufficient recognition (and selectivity) with respect to the molecularly imprinted polymer [4, 11-15]. Here, the monomeric hGH and dimeric hGH were used for the entrapment and recognition. Typically, polyacrylamide gels are selective because the size and shape of the cavity originally occupied by the substrate is retained by high crosslinking density, but in case of Hjertén's method this does not apply [10]. In case of Hjertén's method the degree of crosslinking of the hydrogel is much lower than that used in conventional molecular imprinting. Many experiments with protein templates and various functional monomers show that the incorporation of functional groups with either negative or positive charge into the polyacrylamide gel leads also to high selectivity for the recognition of the protein [12-15]. In our previous work [13] for the first time it was shown that monomeric and dimeric hGH can successfully be molecularly imprinted into polyacrylamide hydrogels with ionic functional groups (and dimeric hGH was molecularly imprinted for the first time). Significant imprinting efficiency was detected using ultraviolet absorbance. The selectivity study shows excellent results for selective binding when the analyte and the template had been identical and a very low binding of other proteins. Natural antibodies usually only reflect the concentration of hGH and do not discriminate the structural differences between hGH dimer and monomer $[1,15]$.

Ghasemzadeh and coworkers $[14,15]$ used a molecular imprinting approach to synthesize artificial gel antibodies against hGH dimer and monomer. The gel antibodies could specifically adsorb these growth hormone forms used for their preparation. It was concluded that these two sets of artificial gel antibodies might be useful for discriminating between dimeric and monomeric hGH in clinical samples. 
So far, no investigation of the molecular imprinting of bigger (oligomeric) molecular forms of hGH exists.

The aim of this work was the synthesis of molecularly imprinted polyacrylamide hydrogels with or without ionizable groups in the presence of the tetrameric form of hGH. Furthermore, the binding of the template hormone, other molecular forms of the hormone and other proteins to the imprinted polymer was investigated.

Such molecularly imprinted polymers have possible future applications in the fields of quantitative sorbent assays (as artificial antibodies) or chromatography.

\section{Experimental}

\subsection{Materials}

The monomeric human growth hormone with the molecular weight of $22 \mathrm{kDa}$, dimeric and oligomeric (three-five globular units) molecular forms of hGH were prepared and characterized as described earlier [16]. Bovine serum albumine, $\geq 99 \%$ (A7638, Sigma, Saint Louis, USA), human serum albumin, $\geq 99 \%$ (A3782, Sigma, Saint Louis, USA), lysozyme (from chicken egg white), $\geq 98 \%$ (L4919, Sigma, Saint Louis, USA ), acrylamide, $\geq 99 \%$, (A3553, Sigma, Saint Louis, USA), 2-(dimethylamino)-ethylmethacrylate, $\geq 98 \%$ (234907, Aldrich, Saint Louis, USA), methacrylic acid, $\geq 99 \%$ (155721, Aldrich, Saint Louis, USA ), $N, N^{\prime}$-methylene bisacrylamide, $\geq 99 \%$, (101546, Merck, Darmstadt, Germany), $N, N, N^{\prime}, N^{\prime}$ tetramethylethylenediamine (1610801, BioRad, Hercules, USA), potassium persulfate, $99.99 \%$ (379824, Aldrich, Saint Louis, USA), sodium chloride, $\geq 99.5 \%$ (S7653, Sigma-Aldrich, Saint Louis, USA), sodium dodecyl sulfate (SDS) $\geq 98.5 \%$ (L3771, Sigma, Saint Louis, USA), $1 \mathrm{M} \mathrm{NaOH}$ solution (43617, SigmaAldrich, Saint Louis, USA) were used as obtained.

Deionized water was produced by a Millipore water system. PBS buffer solution ( $\mathrm{pH}$ 6.2) was prepared from $18.4 \mathrm{~mL}$ disodium hydrogen phosphate $1 / 15 \mathrm{M}$ solution (106587, Merck, Darmstadt, Germany) and $81.6 \mathrm{~mL}$ potassium dihydrogen phosphate $1 / 15 \mathrm{M}$ solution (104875, Merck, Darmstadt, Germany).

\subsection{Instruments}

Ultraviolet absorbance (by $280 \mathrm{~nm}$ ) measurements were performed using a spectrophotometer Genesys 10S UV-Vis (840-208100, Thermo Scientific ${ }^{\mathrm{TM}}$, USA). Every measurement was repeated three times.

\subsection{Preparation of molecularly imprinted polymers by Hjertén's method (MIP-H)}

In a typical polymer synthesis PBS buffer solution (1/15 M, pH 6.2) was used. Acrylamide $0.288 \mathrm{~g}$ $\left(4.01 \cdot 10^{-3} \mathrm{~mol}\right)$, various amount of $N, N^{\prime}$-methylene bisacrylamide (MBA) (was varied from $0.006 \mathrm{~g}$ $\left(3.853 \cdot 10^{-5} \mathrm{~mol}\right)$ up to $\left.0.012 \mathrm{~g}\left(7.705 \cdot 10^{-5} \mathrm{~mol}\right)\right)$ and hGH $0.015 \mathrm{~g}$ (tetrameric form of hGH or c.a. 1:1:1 mixture of trimeric, tetrameric and pentameric forms of hGH) were mixed with $5 \mathrm{~mL}$ of PBS in a Schlenk tube at $25^{\circ} \mathrm{C}$ and purged with nitrogen for 15 min. After purging, potassium persulfate [5\% (w/w) aqueous solution] $0.05 \mathrm{~mL}$ and TEMED [5\% $(\mathrm{w} / \mathrm{w})$ aqueous solution] $0.05 \mathrm{~mL}\left(3.23,10^{-5} \mathrm{~mol}\right)$ was added to the solution to start the polymerization. After the polymerization proceeded for $30 \mathrm{~min}$, the solution mixture was wet-sieved through a 100 mesh sieve. The resultant gels (MIP-H-tetra in case of tetrameric form of hGH or MIP-H-mix in case of mixture of trimeric, tetrameric and pentameric forms of hGH) were washed with $200 \mathrm{~mL}$ deionized water three times, $200 \mathrm{~mL} \mathrm{10 \%} \mathrm{acetic} \mathrm{acid} \mathrm{and} \mathrm{2 \%} \mathrm{SDS} \mathrm{so-}$ lution three times, $200 \mathrm{~mL}$ deionized water three times. UV spectra for the wash liquid were taken to determine the amount of hGH extracted. The gels were lyophilized and sieved to obtain particles of $100-150 \mu \mathrm{m}$ size which were stored at ambient temperature.

\subsection{Preparation of molecularly imprinted polymers with ionized functional groups (MIP)}

In a typical polymer synthesis, 2-(dimethylamino) ethyl methacrylate $0.4824 \mathrm{~mL}\left(2.805 \cdot 10^{-3} \mathrm{~mol}\right)$ and methacrylic acid $0.2428 \mathrm{~mL}\left(2.834 \cdot 10^{-3} \mathrm{~mol}\right)$ were mixed with $3.55 \mathrm{~mL}$ of PBS buffer solution (1/15 M, $\mathrm{pH}$ 6.2). The solution was adjusted to $\mathrm{pH} 6.2$ by $1 \mathrm{M}$ $\mathrm{NaOH}$. Then, acrylamide $0.2035 \mathrm{~g}\left(2.834 \cdot 10^{-3} \mathrm{~mol}\right)$, various amount of $N, N^{\prime}$-methylene bisacrylamide (MBA) (was varied from $0.05 \mathrm{~g}\left(3.21 \cdot 10^{-4} \mathrm{~mol}\right)$ up to $\left.0.1 \mathrm{~g}\left(6.42 \cdot 10^{-4} \mathrm{~mol}\right)\right)$ and $\mathrm{hGH}$ form $0.06 \mathrm{~g}$ $\left(2.727 \cdot 10^{-6} \mathrm{~mol}\right)$ were added to the solution in a Schlenk tube at $25^{\circ} \mathrm{C}$ and purged with nitrogen for $15 \mathrm{~min}$. The concentration of hGH (tetrameric form of hGH or mixture (c.a. 1:1:1) of trimeric, tetrameric and pentameric forms of hGH) in the solution was $1.29 \%(\mathrm{w} / \mathrm{w})$. After purging, potassium persulfate $[1.5 \% \quad(\mathrm{w} / \mathrm{w})$ aqueous solution] $0.25 \mathrm{~mL}$ $\left(1.39 \cdot 10^{-5} \mathrm{~mol}\right)$ and TEMED $[3.75 \%(\mathrm{w} / \mathrm{w})$ aqueous solution] $0.1 \mathrm{~mL}\left(3.23 \cdot 10^{-5} \mathrm{~mol}\right)$ was added to the 
solution to start the polymerization. After the polymerization proceeded for $30 \mathrm{~min}$, the solution mixture was wet-sieved through a 100 mesh sieve. The resultant gels (MIP-tetra in case of tetrameric form of hGH or MIP-mix in case of mixture of trimeric, tetrameric and pentameric forms of hGH) were washed with $200 \mathrm{~mL}$ deionized water three times, $200 \mathrm{~mL} \mathrm{NaCl}$ solution $(0.1 \mathrm{M})$ three times and $200 \mathrm{~mL}$ deionized water three times. UV spectra for the wash liquid were taken to determine the amount of hGH extracted. The gels were lyophilized and sieved to obtain particles of 100-150 $\mu \mathrm{m}$ size which were stored at ambient temperature.

\subsection{Investigation of molecularly imprinted polymers}

After extraction of the template the hGH-tetramerimprinted gel particles were added to a solution in which a certain amount of hGH tetramer $(50 \%$ of the amount of template removed from MIP) was dissolved. The amount of the lyophilized gel was $0.05 \pm 0.001 \mathrm{~g}$. The concentration of hGH (tetramer and other molecular forms of hGH) or other test-proteins in test solution was $0.129 \%(\mathrm{w} / \mathrm{w})$. The liquid supernatant was separated from the gel after the test tube was centrifuged at $1000 \mathrm{rpm}$ during $5 \mathrm{~min}$. All experiments were reproduced three times.

\section{Results and discussion}

\subsection{Synthesis of the imprinted polymers}

The imprinted functionalized polyacrylamide hydrogels (MIP) were prepared by free radical polymerization of acrylamide, 2-(dimethylamino)ethyl methacrylate and methacrylic acid (1:1:1 ratio) together with the crosslinker $N, N^{\prime}$-methylene bisacrylamide in buffer solution in the presence of the selected molecular form of the hGH. In case of Hjertén's method (MIP-H) only acrylamide and crosslinker $N, N^{\prime}$-methylene bisacrylamide was used. A highly effective redox initiation system was used to allow polymerization by both methods at the same room temperature for the same short time ( $30 \mathrm{~min}$.). The template is enough stable under these conditions [1].

\subsection{The removal of the template}

After the polymerization was completed, the template had to be removed by intensive washing. The volume change in the MIP hydrogel is very dependent on the ion strength of the solution [13]. To remove the template molecules from the MIP as effectively as possible, the MIP particles were rinsed therefore by changing the ion strength of the solution. The removal of the template from the imprinted particles was confirmed by quantitative measurements of the UV absorbance at $280 \mathrm{~nm}$ of the $\mathrm{NaCl}$ solution used for washing the hGH from the particles. A part of template (approximately $40-50 \%$ in case of MIP-Htetra and $30-40 \%$ in case of MIP-tetra) could not be extracted from the gel and thus, was fixed to MIP (Table 1). Increasing of intensity of washing procedure cannot solve this problem. As possible reasons for that one can assume that some hGH molecules are entrapped so strongly in the cross-linked hydrogel that it could not be removed. The second possible reason - some free radicals attacked these hGH molecules leading to covalent binding to the gel - can be excluded because of previous experience with imprinting of monomeric and dimeric hGH forms [13]. The amount of template which could not be extracted from the gel (MIP-tetra) was significantly higher than in the case of imprinting experiments of monomeric and dimeric hGH (it was only 9-10\%) [13] using the same polymerization conditions. This can be explained by the complicated and not so rigid structure of this hGH form.

A big part of mixture-template (approximately $75 \%$ in case of MIP-H-mix and $70 \%$ in case of MIP-mix) could not be extracted from the gel. MIPs prepared with the mixture of three molecular forms of hGH as template cannot be considered as potential artificial antibodies against molecular forms of hGH. On the other hand, these results (and the results obtained in our previous work [13]) can show us that only one single molecular form of hGH can serve as possible template for molecular imprinting.

Table 1. Efficiency of the template removal from the prepared MIPs

\begin{tabular}{|l|c|c|c|}
\hline \multicolumn{1}{|c|}{ MIP } & $\begin{array}{c}\text { Amount of MBA } \\
\text { in polymerization } \\
\text { mixture } \\
\text { [g] }\end{array}$ & $\begin{array}{c}\text { From MIP } \\
\text { extracted } \\
\text { template in } \\
{[\%]}\end{array}$ & $\begin{array}{c}\text { Fixed to MIP } \\
\text { template in } \\
{[\%]}\end{array}$ \\
\hline MIP-H-tetra & $0.006 \pm 0.0001$ & $60.2 \pm 0.5$ & $39.8 \pm 0.5$ \\
MIP-H-tetra & $0.009 \pm 0.0001$ & $59.1 \pm 0.4$ & $40.9 \pm 0.4$ \\
MIP-H-tetra & $0.012 \pm 0.0001$ & $50.1 \pm 0.5$ & $59.9 \pm 0.5$ \\
MIP-H-mix & $0.006 \pm 0.0001$ & $24.2 \pm 0.3$ & $75.8 \pm 0.3$ \\
MIP-H-mix & $0.009 \pm 0.0001$ & $24.9 \pm 0.3$ & $75.1 \pm 0.3$ \\
MIP-H-mix & $0.012 \pm 0.0001$ & $23.9 \pm 0.4$ & $76.1 \pm 0.4$ \\
MIP-tetra & $0.05 \pm 0.0001$ & $70.2 \pm 0.6$ & $29.8 \pm 0.6$ \\
MIP-tetra & $0.075 \pm 0.0001$ & $69.5 \pm 0.5$ & $30.5 \pm 0.5$ \\
MIP-tetra & $0.1 \pm 0.0001$ & $60.1 \pm 0.5$ & $39.9 \pm 0.5$ \\
MIP-mix & $0.05 \pm 0.0001$ & $30.6 \pm 0.5$ & $69.4 \pm 0.5$ \\
MIP-mix & $0.075 \pm 0.0001$ & $29.9 \pm 0.4$ & $70.1 \pm 0.4$ \\
MIP-mix & $0.1 \pm 0.0001$ & $28.9 \pm 0.5$ & $71.1 \pm 0.5$ \\
\hline
\end{tabular}




\subsection{Recognition of hGH templates}

After extraction of the template the imprinted gel (tetrameric hGH as template and Hjertén's method of imprinting) particles were added to a solution in which a certain amount $(50 \%$ of the amount of template removed from MIP) of test-hGH-form (or its mixture of trimeric, tetrameric and pentameric hGH) was dissolved. The amount of the lyophilized gel was $0.05 \mathrm{~g}$. The concentration of hGH in test solution was $0.129 \%(\mathrm{w} / \mathrm{w})$. The liquid supernatant was separated from the gel after the test tube was centrifuged at $1000 \mathrm{rpm}$ during $5 \mathrm{~min}$. The amount of hGH adsorbed onto the gel particles was calculated by subtracting the quantity of hGH in the supernatant from that in the original solution. The same experiment was carried out with MIP with ionized functional groups using the test-hGH form or its mixture.

Non-imprinted polymers (NIPs) were synthesized the same way as the MIP-H-tetra or MIP-tetra (by all three different concentrations of MBA in polymerization mixture) and after polymerization the NIPs were treated exactly the same way as the appropriate MIPs. The non-specific binding of hGH (tetrameric hGH or mixture of three molecular forms of hGH) to the NIP particles was also measured and found to be only $1.0 \pm 0.1 \%$ for polymerization by Hjertén's method and $0.9 \pm 0.1 \%$ for polymerization

Table 2. Specifically bound amount of hGH $(B)$ on the MIPs prepared by Hjertén's method (the same test protein (or mixture) was used as the template)

\begin{tabular}{|c|c|c|c|}
\hline MIP & $\begin{array}{c}\text { Amount of MBA in } \\
\text { polymerization } \\
\text { mixture } \\
\text { [g] }\end{array}$ & $\begin{array}{c}\boldsymbol{B} \text { after 2 h } \\
{[\mathbf{\%}]}\end{array}$ & $\begin{array}{c}\boldsymbol{B} \text { after } \mathbf{1 8} \mathbf{~ h} \\
{[\mathbf{\%}]}\end{array}$ \\
\hline MIP-H-tetra & $0.006 \pm 0.0001$ & $40.3 \pm 0.5$ & $49.7 \pm 0.5$ \\
MIP-H-tetra & $\mathbf{0 . 0 0 9} \pm \mathbf{0 . 0 0 0 1}$ & $\mathbf{4 9 . 1} \pm \mathbf{0 . 4}$ & $\mathbf{5 4 . 9} \pm \mathbf{0 . 5}$ \\
MIP-H-tetra & $0.012 \pm 0.0001$ & $42.1 \pm 0.5$ & $50.4 \pm 0.5$ \\
MIP-H-mix & $0.006 \pm 0.0001$ & $5.2 \pm 0.2$ & $5.8 \pm 0.3$ \\
MIP-H-mix & $0.009 \pm 0.0001$ & $5.4 \pm 0.2$ & $5.6 \pm 0.2$ \\
MIP-H-mix & $0.012 \pm 0.0001$ & $5.0 \pm 0.3$ & $5.4 \pm 0.3$ \\
\hline
\end{tabular}

Table 3. Specifically bound amount of hGH $(B)$ on the MIPs with ionized functional groups

\begin{tabular}{|l|c|c|c|}
\hline MIP & $\begin{array}{c}\text { Amount of MBA in } \\
\text { polymerization } \\
\text { mixture } \\
\text { [g] }\end{array}$ & $\begin{array}{c}\boldsymbol{B} \text { after 2 h } \\
{[\mathbf{\%}]}\end{array}$ & $\begin{array}{c}\boldsymbol{B} \text { after 18 h } \\
{[\mathbf{\%}]}\end{array}$ \\
\hline MIP-tetra & $0.05 \pm 0.0001$ & $38.4 \pm 0.5$ & $43.6 \pm 0.5$ \\
MIP-tetra & $\mathbf{0 . 0 7 5} \pm \mathbf{0 . 0 0 0 1}$ & $\mathbf{4 5 . 2} \pm \mathbf{0 . 4}$ & $\mathbf{5 0 . 9} \pm \mathbf{0 . 4}$ \\
MIP-tetra & $0.1 \pm 0.0001$ & $40.2 \pm 0.4$ & $46.4 \pm 0.5$ \\
MIP-mix & $0.05 \pm 0.0001$ & $6.6 \pm 0.4$ & $7.4 \pm 0.4$ \\
MIP-mix & $0.075 \pm 0.0001$ & $5.9 \pm 0.4$ & $7.1 \pm 0.4$ \\
MIP-mix & $0.1 \pm 0.0001$ & $5.8 \pm 0.4$ & $6.8 \pm 0.3$ \\
\hline
\end{tabular}

of MIPs with ionized functional groups (by all three different concentrations of MBA in polymerization mixture).

Similar low non-specific binding of hGH was found in our previous experiments with monomeric and dimeric forms of hGH and NIP with ionized functional groups [13]. By subtracting the amount of hGH adsorbed onto the non-imprinted reference gel particles from that adsorbed onto the hGH-imprinted polymer particles, the amount of specifically bound hGH (B) was calculated and transferred to $\%$ from original concentration values (Table 2 and Table 3 ). All MIPs prepared with the mixture of three molecular forms of hGH as template show very low value of specifically binding $B$. The MIPs prepared using single molecular form of hGH (tetramer) show much higher $B$ value. It shows that single molecular form (as template) can give MIPs with good specific binding.

\subsection{Selectivity of the imprinted polymers}

The MIPs with best recognition of template protein was used for the investigation of cross-reactivity of the MIPs with other hGH forms and other proteins. To confirm the selectivity of the imprinted polymer, monomeric and dimeric molecular forms of hGH and other proteins such as bovine serum albumin (BSA), human serum albumin (HSA), or lysozyme (LSZ) were dissolved in the solutions, and the same experiments as those for the template hGH form were carried out using the same concentration of the protein. Table 4 represents the results of investigation of the adsorption onto MIPs after $18 \mathrm{~h}$ in $\%$ from the original solution.

The other proteins, however, showed a very low tendency to bind to the MIPs with values in the range of $0.5-1.6$. Two of the weakly adsorbing proteins - BSA and HSA - have approximately the same isoelectric

Table 4. Selectivity of the imprinted polymers MIP-H-tetra and MIP-tetra in adsorption experiments using different analytes

\begin{tabular}{|c|c|c|c|}
\hline \multicolumn{2}{|c|}{ Test protein } & \multicolumn{2}{|c|}{$\begin{array}{c}\text { Adsorption onto MIP after } \\
18 \mathrm{~h} \text { from the original } \\
\text { solution } \\
{[\%]}\end{array}$} \\
\hline hGH form & Other protein & MIP-H-tetra & MIP- tetra \\
\hline- & BSA & $0.9 \pm 0.1$ & $1.1 \pm 0.1$ \\
\hline - & HSA & $1.6 \pm 0.2$ & $1.4 \pm 0.1$ \\
\hline- & LSZ & $0.5 \pm 0.1$ & $0.6 \pm 0.1$ \\
\hline tetrameric $\mathrm{hGH}$ & - & $54.9 \pm 0.5$ & $50.9 \pm 0.4$ \\
\hline dimeric $\mathrm{hGH}$ & - & $5.5 \pm 0.1$ & $6.4 \pm 0.1$ \\
\hline monomeric hGH & - & $3.9 \pm 0.1$ & $5.1 \pm 0.1$ \\
\hline
\end{tabular}


point as hGH (pH 5.1 or 5.2). This can be considered as a good proof for an effective imprinting mechanism and not just plain adsorption capacity of the hydrogels. The cross-reactivity of the monomeric hGH (up to $5.1 \pm 0.1$ ) and dimeric hGH (up to $6.4 \pm 0.1$ ) to the MIPs is low enough. These MIPs can be considered as artificial antibodies against tetrameric form of hGH. Such molecularly imprinted hydrogels can provide selective recognition of this molecular form of human growth hormone and, thus, solve problems related to the absence of the natural antibodies that can specifically recognize this molecular form of hGH. Furthermore, the proposed imprinted polymers have general advantages as compared to immobilized natural antibodies like robustness and longer lifetime.

\section{Conclusions}

The tetrameric molecular form of the human growth hormone was molecularly imprinted into polyacrylamide hydrogels with or without ionic functional groups. A part of template (approximately $40-50 \%$ in case of hydrogel without ionic functional groups and $30-40 \%$ in case of other hydrogel) could not be extracted from the gel. The hydrogels with best recognition of the template showed selective binding when the analyte and the template had been identical and a very low binding of other proteins. The monomeric and dimeric molecular forms of hGH showed low cross-reactivity within the imprinted hydrogels. Thus, the reported polyacrylamide hydrogels can be considered as artificial antibodies against the tetrameric form of the hormone.

\section{Acknowledgements}

The research was supported in part by Alexander von Humboldt-Stiftung.

\section{References}

[1] Popii V., Baumann G.: Laboratory measurement of growth hormone. Clinica Chimica Acta, 350, 1-16 (2004).

https://doi.org/10.1016/j.ccen.2004.06.007

[2] Vasapollo G., Del Sole R., Mergola L., Lazzoi M. R., Scardino A., Scorrano S., Mele G.: Molecularly imprinted polymers: Present and future prospective. International Journal of Molecular Sciences, 12, 5908-5945 (2011).

https://doi.org/10.3390/ijms12095908
[3] Herrero E. P., Del Valle E. M. M., Peppas N. A.: Protein imprinting by means of alginate-based polymer microcapsules. Industrial and Engineering Chemistry Research, 49, 9811-9814 (2010). https://doi.org/10.1021/ie101068z

[4] Kryscio D. R., Peppas N. A.: Critical review and perspective of macromolecularly imprinted polymers. Acta Biomaterialia, 8, 461-473 (2012). https://doi.org/10.1016/j.actbio.2011.11.005

[5] Cai D., Ren L., Zhao H., Xu C., Zhang L., Yu Y., Wang H., Lan Y., Roberts M. F., Chuang J. H., Naughton M. J., Ren Z., Chiles T. C.: A molecular-imprint nanosensor for ultrasensitive detection of proteins. Nature Nanotechnology, 5, 597-601 (2010). https://doi.org/10.1038/nnano.2010.114

[6] Bacskay I., Takátsy A., Végvári Á., Elfwing A., Ballagi-Pogdány A., Kilár F., Hjertén S.: Universal method for synthesis of artificial gel antibodies by the imprinting approach combined with a unique electrophoresis technique for detection of minute structural differences of proteins, viruses, and cells (bacteria). III: Gel antibodies against cells (bacteria). Electrophoresis, 27, 4682-4687 (2006) https://doi.org/10.1002/elps.200600192

[7] Zahedi P., Ziaee M., Abdouss M., Farazin A., Mizaikoff B.: Biomacromolecule template-based molecularly imprinted polymers with an emphasis on their synthesis strategies: A review. Polymers Advanced Technologies, 27, 1124-1142 (2016). https://doi.org/10.1002/pat.3754

[8] Maier N. M., Lindner W.: Chiral recognition applications of molecularly imprinted polymers: A critical review. Analytical and Bioanalytical Chemistry, 389, 377397 (2007).

https://doi.org/10.1007/s00216-007-1427-4

[9] Liao J-L., Wang Y., Hjertén S.: A novel support with artificially created recognition for the selective removal of proteins and for affinity chromatography. Chromatographia, 42, 259-262 (1996).

https://doi.org/10.1007/BF02290306

[10] Hjertén S., Liao J-L., Nakazato K., Zamaratskaia G., Zhang H-X.: Gels mimicking antibodies in their selective recognition of proteins. Chromatographia, 44, 227 234 (1997).

https://doi.org/10.1007/BF02466386

[11] Ghasemzadeh N., Nyberg F., Hjertén S.: Highly selective artificial gel antibodies for detection and quantification of biomarkers in clinical samples. II. Albumin in body fluids of patients with neurological disorders. Journal of Separation Science, 31, 3954-3958 (2008). https://doi.org/10.1002/jssc.200800386

[12] Tong D., Heényi C., Bikádi Z., Gao J. P., Hjertén S.: Some studies of the chromatographic properties of gels ('artificial antibodies/receptors') for selective adsorption of proteins. Chromatographia, 54, 7-14 (2001). https://doi.org/10.1007/BF02491825 
[13] Kublickas R., Werner C., Jarienė G., Voit B., Lašas L.: Polyacrylamide gels containing ionized functional groups for the molecular imprinting of human growth hormone. Polymer Bulletin, 58, 611-617 (2007).

https://doi.org/10.1007/s00289-006-0694-0

[14] Ghasemzadeh N., Rossbach U. L., Johansson B-M., Nyberg F.: Application of artificial gel antibodies for investigating molecular polymorphisms of human pituitary growth hormone. Amino Acids, 40, 1249-1255 (2011).

https://doi.org/10.1007/s00726-011-0840-3

[15] Ghasemzadeh N., Wilhelmsen T. W., Nyberg F., Hjertén S.: Precautions to improve the accuracy of quantitative determinations of biomarkers in clinical diagnostics. Electrophoresis, 31, 2722-2729 (2010).

https://doi.org/10.1002/elps.201000243
[16] Kublickas R., Jarienè G., Lašas L.: Investigation of the monomeric and oligomeric molecular forms of human growth hormone and poly-L-lysine using fluorescence anisotropy measurement method. Polymer Bulletin, 56, 193-199 (2006).

https://doi.org/10.1007/s00289-005-0474-2

[17] Zhu W., Ding J.: Synthesis and characterization of a redox-initiated, injectable, biodegradable hydrogel. Applied Polymer Science, 99, 2375-2383 (2006). https://doi.org/10.1002/app.22674

[18] Davies M. P., De Biasi V., Perrett D.: Approaches to the rational design of molecularly imprinted polymers. Analytica Chimica Acta, 504, 7-14 (2004).

https://doi.org/10.1016/S0003-2670(03)00812-2 